\title{
Workflow for Correlatively Imaging Mouse Pulmonary Valve Extracellular Matrix
}

Yifei Liu ${ }^{1}$, David W. McComb ${ }^{1}$, Yong-Ung Lee ${ }^{2}$ and Christopher K. Breuer ${ }^{1,2}$

${ }^{1}$ Center for Electron Microscopy and Analysis, The Ohio State University, Columbus, $\mathrm{OH}$.

2. Nationwide Children's Hospital, Department of Tissue Engineering, Columbus, OH.

A human pulmonary heart valve opens and closes around 3 billion times over a lifetime and is responsible for the unidirectional flow of blood [1]. It is a purely biomechanical structure that responds to the external hemodynamic environment. Even though the mouse model is advantageous because of the ability to produce a large identical population, the mouse pulmonary valve is vastly understudied because of the surgical and instrumental demands. The pulmonary valve is composed of three distinct layers, the fibrosa, spongiosa and ventricularis; each compositionally unique and well-studied [2]. However, the reorganization of the extracellular matrix to accommodate large strains has yet to be elucidated. Collagen I is a fibrillar extracellular matrix protein that is responsible for the mechanical strength [3]. Collagen I has a characteristic banding structure of $67 \mathrm{~nm}$ and its disorganization have been linked to congenital disease and defective heart functions [4,5]. To characterize the hierarchical organization of the extracellular collagen from single fiber to tissue level, multiple length scales need to be traversed. We propose a correlative workflow utilizing micro-computed tomography $(\mu \mathrm{CT})$, scanning electron microscopy and transmission electron microscopy (TEM) to capture the three-dimensional collagen organization within the spatially and temporally varying heart valve.

Mice were anesthetized with a solution of ketamine/xylazine and sacrificed by exsanguination. The heart was excised and pressurized from the arterial side of the pulmonary valve with a glutaraldehyde/paraformaldehyde fixture at physiological pressure. Applying transvalvular pressures gives varying degrees of pulmonary valve opening (Figure 1). The whole heart was trimmed to an approximately $3 \times 3 \times 3 \mathrm{~mm}$ block, isolating the pulmonary valve and immediate surrounding tissue. Specimens were stained using a suite of osmium tetroxide, uranyl acetate, and lead aspartate. Dehydration by sequential treatments of ethanol and embedment in resin followed.

During sample processing, the orientation of the pulmonary valve is lost and to reobtain that information, embedded specimens were initially imaged using $\mu \mathrm{CT}$. The purpose of this scan is two-fold: a volume rendering of the pulmonary valve to degree of closure, and to serve as a map for further sample processing. From the guidance of the $\mu \mathrm{CT}$, the precise coordinates of the pulmonary valve were known, and specimens were sliced to the location of the heart valve using microtomy. Figure 2 shows a $\mu \mathrm{CT}$ volume rendering of an excised heart tissue with the pulmonary valve. A strong visual correction between the virtual slices of the $\mu \mathrm{CT}$ scan and the physical specimen block is shown in the right column of Figure 2.

Once at a region of interest, the specimen block can be used for serial block face sectioning for higher resolution volume rendering and/or TEM. Typically, TEM gives limited information about organization because the output is a $2 \mathrm{D}$ projection. Since we have the corresponding $\mu \mathrm{CT}$ data, we know exactly the origin of the TEM specimen and can relate that back to an anatomical axis, providing insight to the underlying biomechanics of the valve. 
References:

[1] MS Sacks, DB Smith, and ED Hiester, Journal of Biomedical Materials Research 41.1 (1998), p. 131.

[2] Schoen, FJ., Cardiovascular Pathology 14.4 (2005), p. 189.

[3] Fratzl, P. et al, Journal of Structural Biology 122.1-2 (1998), p. 119.

[4] Hinton, RB et al, Circulation Research 98.11 (2006), p. 1431.

[5] Hinton, Robert B. and Katherine E. Yutzey. Annual Review of Physiology 73 (2011), p. 29.

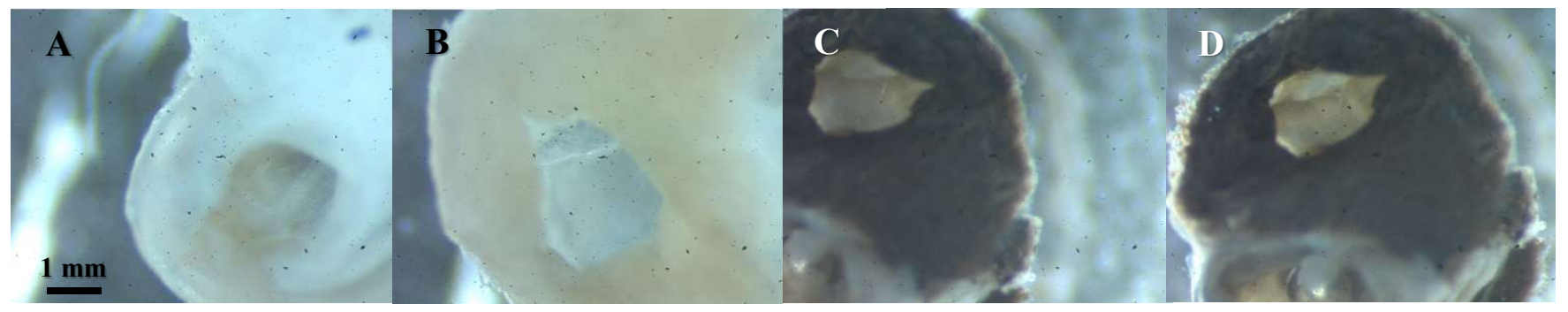

Figure 1. (A and B) Optical microscope images of pulmonary valve following fixation and (C and D) osmium staining from the (A and C) pulmonary arterial side and (B and D) ventricular side. Valves were fixed with glutaraldehyde and paraformaldehyde at $20 \mathrm{mmHg}$ transvalvular pressure. Pulmonary valve shows a closed configuration and no visible signs of shrinking after osmium processing.
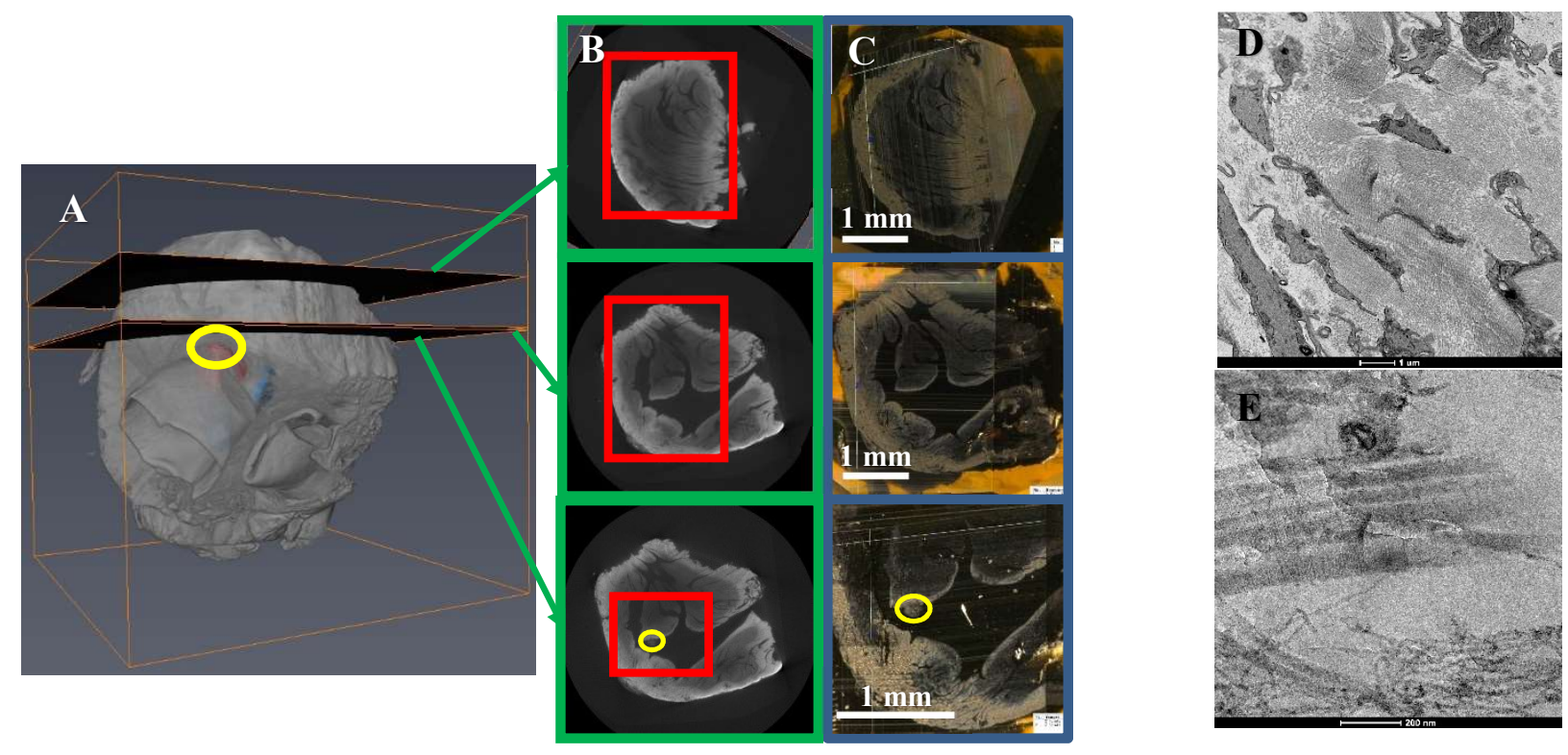

Figure 2. (A) Micro-computed tomographic volume rendering of pulmonary heart valve at $20 \mathrm{mmHg}$. Comparison of (B) virtual slices to (C) sample block cross section shows strong visual correlation. Yellow circle indicates identical regions across three images. (D and E) TEM images taken from yellow circled region showing collagen packing and banding structure. 